\title{
A GENERALIZATION OF LUCAS' THEOREM TO VECTOR SPACES
}

\author{
NEYAMAT ZAHEER \\ Mathematics Department \\ King Saud University \\ P.O. Box 2455 \\ Riyadh 11451, Saudi Arabia
}

(Received June 2, 1988 and in revised form August 15, 1991)

\section{ABSTRACT.}

The classical Lucas' theorem on critical points of complex-valued polynomials has been generalized (cf. [1]) to vector-valued polynomials defined on $K$-inner product spaces. In the present paper, we obtain a generalization of Lucas' theorem to vector-valued abstract polynomials defined on vector spaces, in general, which includes the above result of the author [1] in $K$-inner product spaces. Our main theorem also deduces a well-known result due to Marden on linear combinations of polynomial and its derivative. At the end, we discuss some examples in support of certain claims.

KEY WORDS AND PHRASES: Abstract polynomials and their pseudo-derivatives, (supergeneralized) circular regions, $K$-inner product spaces.

1991 AMS SUBJECT CLASSIFICATION CODES. Primary 30C10; Secondary 30C15.

\section{INTRODUCTION.}

Throughout, unless mentioned otherwise, $E$ and $V$ denote vector spaces over an algebraically closed field of characteristic zero and $P^{*}$ denotes the family of all nonconstant polynomials $P: E \rightarrow V$. The concept of Lucas-sets for the family $P^{*}$, when $E$ is a $K$-inner product space, was introduced in [1] and it was shown that every member $A$ of the family $D\left(E_{\omega}\right)$ of all generalized circular regions of $E_{\omega}$, with $\omega \notin A$, is a Lucas-set for $P^{*}$. This fact naturally raises two questions: Firstly, does $D\left(E_{\omega}\right)$ exhaust all Lucas-sets in $E_{\omega}$ when $E$ is a $K$-inner product space? Secondly, does there exist an analogous family of Lucas-sets for $p^{*}$ when $E$ is, in general, a vector space? In this paper, we introduce the family $D^{*}\left(E_{\omega}\right)$ of supergeneralized circular regions of $E_{\omega}$ which answers the first question negatively and the second question affirmatively. We employ this family to generalize (to vector-valued abstract polynomials in vector spaces) the classical Lucas' theorem on the zeros of the derivative of a polynomial and a theorem due to Marden on linear combinations of a polynomial and its derivative.

\section{PRELIMINARIES.}

Walsh [2] has shown that the well-known Lucas' theorem (cf. [3] or [4, Theorem $(6,1)]$ ) is equivalent to the following result [4, Theorem (6.2)], namely: Any convex circular region which contains all the zeros of a complex-valued nonconstant polynomial $f$ also contains all the zeros of the derivative $f$ of $f$. In terms of the terminology of Lucas-sets (cf. [1, p. 832]) this result equivalently states that convex circular regions in the complex plane are Lucas-sets for the family of all nonconstant polynomials. Our aim in this paper is to generalize the said Lucas' theorem and to investigate possible Lucas-sets for the family $\vec{P}^{*}$ of all vector-valued abstract polynomials (cf. [1],[5]-[7]) defined on vector spaces $E$ of arbitrary dimension. A detailed analogous study of this problem, in the special case when $E$ is a $K$-inner product space, has already been made in a paper due to the author (cf. [1, pp. 845-847] for a precise statement about Lucas-sets for P). 
The details in the remainder of this section can all be found in [1, pp. 833-835, 839-843], apart from other alternate sources cited for completeness. $E$ and $V$ denote vector spaces of arbitrary dimension over an algebraically closed field $K$ of characteristic zero. We write $E_{\omega}=E \cup\{\omega\}$ and $K_{\infty}=K \cup\{\infty\}$, where $\omega$ (resp. $\infty$ ) is an element having the properties of vector (resp. scalar) infinity (cf. also [8, pp. 352, 372] or [9, p. 116]); $K_{0}$ denotes a maximal ordered subfield of $K$ with $K_{0}$, as the set of all non-negative elements of $K_{0}$, so that (cf. [10, pp. 38-40], [11, p. 56], or [12, pp. 248-255]) $K-K_{0}(i)-\left\{a+i b \mid a, b \in K_{0}\right\}$, where $-i^{2}=1$. Consequently, if $K_{0}=\mathrm{R}$ (the field of reals) then $K-\mathrm{C}$ (the field of complex numbers). For $z \in K$, the definitions of $\bar{z}, \operatorname{Re} z, \operatorname{Im} z$ and $|z|$ are defined as in C. Similarly, the concept of $K$-inner product spaces (briefly written $K$-i.p.s.) $(E,(.,)$.$) and the notions of K_{0}$-convexity and $K_{0}$-normed vector spaces $(E,\|\cdot\|)$ are defined likewise in C (cf. [13, pp. 120-121]). If $(E,\langle.,\rangle$.$) is a K$-i.p.s. then the $K_{0}$-norm on $E$, given by $\|x\|=\langle x, x\rangle^{1 / 2}$ for $x \in E$, defines for each $b \in E$ the mapping

$$
\Psi_{b}(x)=(x-b) /\|x-b\|^{2} \quad \forall \quad x \in E
$$

(with the tacit assumption that $x /\|x\|^{2}$ equals $\omega$ or 0 according as $x$ is 0 or $\omega$ ) which, in turn, defines the family $D\left(E_{\omega}\right)$ of all generalized circular regions (briefly g.c.r.) of $E_{\omega}[1, \mathrm{pp} .834-835]$. The empty set $\phi$, $E, E_{\omega}$, and the singletons $\{x\}$ (and $E_{\omega}-\{x\}$ ) for $x \in E$ are trivial members of $D\left(E_{\omega}\right)$, whereas the family $B_{8}\left(E_{\omega}\right)$ of all generalized balls is rich in nontrivial members of $D\left(E_{\omega}\right)$.

The concept of abstract homogeneous polynomials is well known (see [4]-[7],[9],[11],[14]-[17]). In what follows we briefly describe abstract polynomials and their pseudo-derivatives. A mapping $P: E \rightarrow V$ is called an abstract polynomial (briefly, a.p.) of degree $n$ if for every $x, y \in E$,

$$
P(x+\rho y)=\sum_{k=0}^{n} A_{k}(x, y) \rho^{k} \quad \forall \rho \in K,
$$

wherein $A_{k}(x, y) \in V$ are independent of $\rho$ and $A_{k}(x, y) \notin 0$. The class of all nth degree a.p.'s is denoted by $P_{n}^{*}$ (or $P_{n}$, if $V=K$ ) and, for $P \in P_{n}^{*}$ given by (2.2), we write

$$
F(P)=\left\{h \in E \mid h=0, A_{n}(0, h)=0\right\} \text {. }
$$

It is known that $F(P) \neq \phi$. Given $P \in P_{n}^{*}$ (via (2.2)) and $h \in F(P)$, we define for each $k=1,2, \ldots, n$, the kth pseudo-derivative $P_{h}^{(k)}$ of $P$ by

$$
P_{h}^{(k)}(x)=k ! A_{k}(x, h) \quad \forall x \in E,
$$

with first few being written as $P_{h}^{\prime}, P_{h}^{\prime \prime}$, etc. It is known (cf. [1, Proposition 2.3 and Remark 2.4 (I)]) that $P_{h}^{(k)} \in P_{n-k}^{*}$ and $h \in F\left(P_{h}^{(k)}\right)$ for all $k$, and that

$$
P_{h}^{(k+1)}(x)=\left(P_{h}^{(k)}\right)_{h}^{\prime}(x) \quad \forall x \in E, 1 \leq k \leq n-1 .
$$

REMARK 2.1 [1, Remark 2.4 (III)]. If $f$ is an (ordinary) polynomial of degree $n$ from $K$ to $K$, then $f$ is an a.p. of degree $n$ from $K$ to $K, F(f)=K-\{0\}$, and

$$
f_{h}^{(k)}(x)=h^{k} f^{(k)}(x) \quad \forall x \in E, \quad h \in K-\{0\},
$$

where $f^{(k)}$ denotes the $k$ th formal derivative of $f($ see $[18$, p. 528], [17, p. 553], or [1, p. 842]). In particular, for $h=1$, we see that $f_{1}^{(k)}-f^{(k)}$ and the two notions coincide. Furthermore, if $K-\mathbf{C}$, then $f_{1}^{(k)}$ becomes precisely the $k$ th derivative $f^{(k)}$ of $f$ as defined via calculus. For $k=1$, we have $f_{1}-f^{\prime}$.

\section{SUPERGENERALIZED CIRCULAR REGIONS}

The study in [1] has revealed that the g.c.r.'s of $E_{\omega}$ and the pseudo-derivatives of a.p.'s from $E$ to $V$, respectively, are natural analogues of (classical) circular regions and derivatives of (ordinary) polynomials in the complex plane, needed to formulate Lucas' theorem in a K-i.p.s. In order to achieve such a breakthrough for vector spaces $E$, in general, one needs to develop an analogous concept of circular regions in a vector space $E$. To this end we introduce in this section the concept of supergeneralized circular regions and establish some general properties and examples for later use. First, we recall the definition of the 
family $D\left(K_{\infty}\right)$ of all generalized circular regions of $K_{\infty}$ as originally introduced by Zervos [8, p. 353]. We say that a subset $A$ of $K_{\infty}$ belongs to $D\left(K_{\infty}\right)$ if and only if either $A$ is one of the sets $\phi, K, K_{\infty}$ or $A$ satisfies the following two conditions: (i) $\theta_{\zeta}(A)$ is $K_{0}$-convex for all $\zeta \in K-A$, where $\theta_{\zeta}(z)=(z-\zeta)^{-1}$ for every $z \in K_{\infty}$ (ii) $\infty \in A$ if $A$ is not $K_{0}$-convex. Fuller details about $D\left(K_{\infty}\right)$ can be found in [18, p. 527-528].

REMARK 3.1. If $E=K$ is taken as a 1-dimensional natural $K$-i.p.s. (cf. [1, Remarks 1.1 and 1.6]), then the family $D\left(E_{\omega}\right)$ in Section 2 (cf. [1, Definition 1.2]) here coincides with the above family $D\left(K_{\infty}\right)$ as introduced by Zervos [8, p. 353]. Thus $\omega$ and $\infty$ can be used interchangeably when $E=K$. That is, $D\left(K_{\infty}\right)$ and $D\left(K_{\omega}\right)$ are equivalent notations.

DEFINITION 3.2. Given $S \subseteq E_{\omega}$, we write

$$
G_{s}(x, y)=\left\{\rho \in K_{\infty} \mid x+\rho y \in S\right\} \quad \forall x, y \in E .
$$

We say that $S \in D^{*}\left(E_{\omega}\right)$ if $G_{s}(x, y) \in D\left(K_{\infty}\right)$ for every $x, y \in E$. Members of $D^{*}\left(E_{\omega}\right)$ are called supergeneralized circular regions (briefly, sg.c.r.) of $E_{\omega}$. Clearly, $\phi, E, E_{\omega}$ are in $D^{\circ}\left(E_{\omega}\right)$ and are called trivial sg.c.r.'s of $E_{\omega}$. Properties of $\omega$ and $\infty$ (cf. [1, p. 834]) imply that (since $G_{S}(x, 0)=K$ or $\phi$ according as $x \in S$ or $x \notin S)$

$$
\infty \notin G_{s}(x, 0) \in D\left(K_{\infty}\right) \quad \forall x \in S
$$

and that

$$
\infty \in G_{s}(x, y) \quad \forall x \in E, y \in E-\{0\} \quad \text { iff } \omega \in S .
$$

Therefore

$$
S \in D^{*}\left(E_{\omega}\right) \text { iff } G_{s}(x, y) \in D\left(K_{\infty}\right) \quad \forall x, y \in E(y \neq 0) .
$$

DEFINITION 3.3 [19, p. 48]. Given an element $(a, \lambda)$ of $E \times(K-\{0\})$, we define the mapping $h_{a, \lambda}: E_{\omega} \rightarrow E_{\omega}$ by

$$
h_{a, \lambda}(z)=a+\lambda z \quad \forall z \in E_{\omega}
$$

and call it a homothetic transformation of $E_{\omega}$. Clearly, $h_{a, \lambda}$ is one-one and onto, and its inverse, $h_{a, \lambda}^{-1}$ is also a homothetic transformation of $E_{\omega}$ given by

$$
h_{a, \lambda}^{-1}(z)=-a \lambda^{-1}+\lambda^{-1} z
$$

PROPOSITION 3.4. Every homothetic transformation of $E_{\omega}$ permutes the family $D^{*}\left(E_{\omega}\right)$.

PROOF. Let $S \in D^{\circ}\left(E_{\omega}\right)$ and $h_{a, \lambda}$ be given by (3.5), so that $h_{a, \lambda}(S)=a+\lambda S-S^{\prime}$ (say). For any $(x, y) \in E^{2}$, we notice that

$$
x+\rho y \in S^{\prime} \text { iff } x_{0}+\rho y_{0} \in S,
$$

where $x_{0}=(x-a) / \lambda$ and $y_{0}=y / \lambda$. Therefore, since $S \in D^{*}\left(E_{\omega}\right)$,

$$
G_{S^{\prime}}(x, y)=g_{s}\left(x_{0}, y_{0}\right) \in D\left(K_{\infty}\right) \forall(x, y) \in E^{2} .
$$

This implies that $S^{\prime}-h_{a, \lambda}(s) \in D^{*}\left(E_{\omega}\right)$. That is,

$$
h_{a, \lambda}\left(D^{*}\left(E_{\omega}\right)\right) \subseteq D^{*}\left(E_{\omega}\right) .
$$

Conversely, to show the reverse containment in (3.6), we take $S^{\prime} \in D^{*}\left(E_{\omega}\right)$ and put $S=h_{a, \lambda}^{-1}\left(S^{\prime}\right)$. Since $h_{a, \lambda}^{-1}$ is also a homothetic transformation of $E_{\omega},(3.6)$ implies that $S \in D^{*}\left(E_{\omega}\right)$. But, then $h_{a, \lambda}(S)=S^{\prime}$ (since $h_{a, \lambda}$ is one-one and onto) and so

$$
D^{*}\left(E_{\omega}\right) \subseteq h_{a, \lambda}\left(D^{*}\left(E_{\omega}\right)\right) .
$$

Now (3.6) and (3.7) complete the proof.

Thus, homothetic transformations play the same role in respect to $D^{*}\left(E_{\omega}\right)$ as do homographic trans- 
formations of $K_{\omega}\left(=K_{\infty}\right)$ (cf. [8, p. 353]) in respect to $D\left(K_{\omega}\right)=D\left(K_{\infty}\right)$. Our next two results say that the family $D^{*}\left(E_{\omega}\right)$ is a natural and coherent extension (to vector spaces) of the family $D\left(E_{\omega}\right)$ in K-i.p.s.'s.

PROPOSITION 3.5. Let $E$ be a K-i.p.s. Then

(a) $D\left(E_{\omega} \subseteq D^{*}\left(E_{\omega}\right)\right.$,

(b) $D\left(E_{\omega}\right)=D^{*}\left(E_{\omega}\right)$ if $\operatorname{dim} E=1$,

(c) $D\left(K_{\omega}\right)=D^{*}\left(K_{\omega}\right)$, if $E=K$ is taken as a 1-dimensional natural $\mathrm{K}$-i.p.s., where $D\left(D_{\omega}\right)=D\left(K_{\infty}\right)$ as in Remark 3.1. Hence $D^{*}\left(\mathbf{C}_{\omega}\right)$ coincides with the family $D\left(\mathbf{C}_{\omega}\right)$ of all g.c.r.'s of $\mathbf{C}_{\omega}$.

PROOF. Given $S \in D\left(E_{\omega}\right)$, consider $G=G_{s}(x, y)$ for any $(x, y) \in E^{2}(y \neq 0)$ in view of (3.4)) as defined in (3.1). To show that $S \in D^{*}\left(E_{\omega}\right)$, we show that $G \in D\left(K_{\infty}\right)$. To this end, we first show that

$$
\theta_{\zeta}(G) \text { is } K_{0} \text {-convex } \forall \zeta \in K-G \text {. }
$$

Choose any $\zeta \in K-G$ (if this is not possible then $G \in D\left(K_{\infty}\right)$ by definition of $D\left(K_{\infty}\right)$ ). Then $b-x+\zeta y \notin S$ and $\psi_{b}(S)$ is $K_{0}$-convex by definition of $D\left(E_{\omega}\right)$ (see (2.1) and [1, p. 834]) such that $\omega \notin \psi_{b}(S)$ (since $\left.b \notin S\right)$. Let $\rho_{i} \in G$ and $t_{i} \in K_{0+}$ with $t_{1}+t_{2}+\ldots+t_{n}=1$ for $i=1,2, \ldots, n$, so that $\infty \times \theta_{\xi}\left(\rho_{i}\right) \in \theta_{\xi}(G)$ and $z_{i}=x+\rho_{i} y \in S$. Since $z_{i}-b=\left(\rho_{i}-\zeta\right) y \neq 0$ for all $i$, we get (cf. (2.1))

$$
\omega \neq \dot{\psi_{b}}\left(z_{i}\right)=\overline{\theta_{\zeta}\left(\rho_{i}\right)} \psi(y) \in \Psi_{b}(S) \text {, }
$$

where $\psi(y)-y /\|y\|^{2} \neq 0($ since $y \neq 0, \omega)$. Since $\psi_{b}(S)$ is $K_{0}$-convex, it implies that

$$
\omega \neq \sum_{i=1}^{n} t_{i} \psi_{b}\left(z_{i}\right)=\sigma \psi(y) \in \psi_{b}(S),
$$

where

$$
\sigma=\sum_{i=1}^{n} t_{i} \overline{\theta_{\xi}\left(\rho_{i}\right)}\left\{\begin{array}{l}
\neq \infty, \\
=0 \text { iff } \omega \in S .
\end{array}\right.
$$

That is, $\sigma \psi(y)=\psi_{b}\left(z_{0}\right)$ for some $z_{0} \in S$. Since $\psi_{b}(z)=\omega$ if and only if $z-\psi(\omega)+b$ (cf. [1, Relation (1.6)]), the properties of $\psi$ in $[1$, p. 834] give

$$
\begin{aligned}
z_{0} & =\psi(\sigma \psi(y))+b, \text { where } \sigma=0 \text { iff } \omega \in S, \\
& =(1 / \bar{\sigma}) y+b=x+(\zeta+1 / \bar{\sigma}) y \in S,
\end{aligned}
$$

so that

$$
\zeta+1 / \bar{\sigma}=\rho_{0} \quad \text { (say) } \in G .
$$

Note that we can take $z_{0}=\omega \in S$ in case $\sigma=0$ and in that case $\rho_{0}=\infty \in G$ due to (3.3). Therefore, in all cases, we have

$$
\bar{\sigma}=\sum_{i=1}^{n} t_{i} \theta_{\zeta}\left(\rho_{i}\right)=1 /\left(\rho_{0}-\zeta\right) \in \theta_{\zeta}(G)
$$

This establishes our claim in (3.8).

Next, we prove that

$$
\infty \in G \text { if } G \text { is not } K_{0} \text {-convex. }
$$

For, if $\infty \notin G$ then $\omega \notin S$ (cf. (3.3)) and $S$ is $K_{0}$-convex as in [1, Definition (1.2)]. Consequently, $G$ must be $K_{0}$-convex by (3.1) and (3.10). Now (3.8) and (3.10) establish that $G \in D\left(K_{\infty}\right)$ for all $x, y \in E(y \propto 0)$. Now the proof of Part (a) is complete in view of (3.4).

(b) The proof of Part (b) follows from that of Part (a) if we let $S \in D^{*}\left(E_{\omega}\right)$ and show that $S \in D\left(E_{\omega}\right)$. Since $\operatorname{dim} E=1$, there exists an element $x_{0} \in E-\{0\}$ such that $E=\left\{\lambda x_{0} \mid \lambda \in K\right\}$ and

$$
G \text { (say) }=G_{s}\left(0, x_{0}\right)-\left\{\rho \in K_{\infty} \mid \rho x_{0} \in S\right\} \in D\left(K_{\infty}\right) \text {. }
$$

Therefore, $x=\rho x_{0} \in S$ if and only if $\rho \in G$. If $b=\zeta x_{0} \notin S$ (so that $\left.\zeta \notin G\right)$ then $\theta_{\zeta}(G)$ is $K_{0}$-convex and 


$$
\begin{aligned}
\Psi_{b}(S) & =\left\{(\rho-\zeta) x_{0} /\left\|(\rho-\zeta) x_{0}\right\|^{2} \mid \rho \in G\right\} \\
& =\left\{\frac{1}{\overline{(\rho-\zeta)}} \cdot \frac{x_{0}}{\left\|x_{0}\right\|^{2}} \mid \rho \in G\right\}, \\
& =\left\{\overline{\theta_{\zeta}(\rho)} \cdot \psi\left(x_{0}\right) \mid \rho \in G\right\}, \\
& =\overline{\theta_{\zeta}(G)} \cdot \psi\left(x_{0}\right) .
\end{aligned}
$$

Hence $K_{0}$-convexity of $\psi_{b}(S)$ follows from the $K_{0}$-convexity of $\theta_{\xi}(G)$ by using Equation (3.11). Next, if $\Psi_{b}(S)$ is not $K_{0}$-convex, then again by $(3.11) \theta_{\zeta}(G)$ is not $K_{0}$-convex and so $\infty \in \theta_{\xi}(G)$ due to the fact that $D\left(K_{\infty}\right)$ is invariant under homographic transformations (cf. [18, Proposition 1.1] or [8, p. 353]). This, together with (3.11) and the fact that $\psi\left(x_{0}\right) \neq \omega$, implies that $\omega \in \psi_{b}(S)$. This would then mean that $b \in S$, which is a contradiction. This goes to establish that $S \in D\left(E_{\omega}\right)$. Part (b) is now established.

(c) If $E-K$ is taken as a 1-dimensional natural K-i.p.s., then $D\left(E_{\omega}\right)$ coincides with $D\left(K_{\omega}\right)=D\left(K_{\infty}\right)$ by Remark 3.1. Therefore, $D\left(K_{\omega}\right)=D^{*}\left(K_{\omega}\right)$ by Part (b), and the proof of Part (c) is complete.

PROPOSITION 3.6. If $E$ is a $\mathrm{K}$-i.p.s. with $\operatorname{dim} E \geq 2$, then

$$
D\left(E_{\omega}\right) \subset D^{*}\left(E_{\omega}\right) \text {. }
$$

PROOF. Let $L(\$ 0)$ be a linear functional from $E$ to $K$. Then

$$
S=\{x \in E \mid L(x)=0\} \subsetneq E .
$$

We show that $S \in D^{*}\left(E_{\omega}\right)-D\left(E_{\omega}\right)$ as follows:

(i) We claim that $S \in D^{*}\left(E_{\omega}\right)$. For $x, y \in E$,

$$
G_{s}(x, y)=\left\{\begin{array}{lll}
\{-L(x) / L(y)\} & \text { if } & L(y) \neq 0, \\
K & \text { if } & L(y)=0=L(x), \\
\phi & \text { if } & L(y)=0 \neq L(x) .
\end{array}\right.
$$

Hence, $G_{s}(x, y) \in D\left(K_{\infty}\right)$ for all $x, y \in E$ and $S \in D^{*}\left(E_{\omega}\right)$.

(ii) We show that $S \notin D\left(E_{\omega}\right)$. Let us first note that $S$ is $K_{0}$-convex and $\omega \notin S$. Choose any element $b \in E-S$ (possible) so that $L(b) \neq 0$. Since $S$ is a maximal subspace of dimension at least one, we can choose distinct elements $x_{1}, x_{2} \in S$, so that $L\left(x_{1}\right)=L\left(x_{2}\right)=0$ and $\psi_{b}\left(x_{1}\right)$ and $\psi_{b}\left(x_{2}\right)$ are distinct elements of $\psi_{b}(S)$. Next, choose

$$
\lambda_{i}-\left\|x_{i}-b\right\|^{2} / D \text { for } i=1,2
$$

where

$$
D=\left\|x_{1}-b\right\|^{2}+\left\|x_{2}-b\right\|^{2} \neq 0, \infty,
$$

so that $\lambda_{1}, \lambda_{2} \in K_{0+}$ with $\lambda_{1}+\lambda_{2}=1$. Therefore,

$$
\lambda_{1} \psi_{b}\left(x_{1}\right)+\lambda_{2} \psi_{b}\left(x_{2}\right)=\left(x_{1}+x_{2}-2 b\right) / D=z \quad \text { (say). }
$$

We claim that $z \notin \Psi_{b}(S)$. For, otherwise, $\psi_{b}(x)=z \approx 0$, $\omega$ for some $x \in S$ and hence $x=z /\|z\|^{2}+b \in S$. But

$$
\begin{aligned}
\frac{z}{\|z\|^{2}}+b & =\frac{x_{1}+x_{2}-2 b}{D} \cdot \frac{D^{2}}{\left\|x_{1}+x_{2}-2 b\right\|^{2}}+b, \\
& =\frac{\left(x_{1}+x_{2}-2 b\right)}{\left\|x_{1}+x_{2}-2 b\right\|^{2}}[D]+b,
\end{aligned}
$$

because $D \neq 0, \infty$ and $x_{1}+x_{2}-2 b \neq 0, \omega$. Therefore, 


$$
\begin{aligned}
0=L(x)=L\left(b+z /\|z\|^{2}\right) & =L(b)+L(z) /\|z\|^{2} \\
& =L(b)+\frac{D}{\left\|x_{1}+x_{2}-2 b\right\|^{2}}\left\{L\left(x_{1}\right)+L\left(x_{2}\right)-2 L(b)\right\} .
\end{aligned}
$$

Since $L\left(x_{1}\right)=L\left(x_{2}\right)=0 \neq L(b)$, we have

$$
\left\|x_{1}+x_{2}-2 b\right\|^{2}=2\left(\left\|x_{1}-b\right\|^{2}+\left\|x_{2}-b\right\|^{2}\right)-\left\|x_{1}+x_{2}-2 b\right\|^{2}+\left\|x_{1}-x_{2}\right\|^{2}
$$

by parallelogram law. That is, $\left\|x_{1}-x_{2}\right\|^{2}=0$, which implies that $x_{1}=x_{2}$. This contradicts the original choice of $x_{1}$ and $x_{2}$. Hence, $z-\lambda_{1} \psi_{b}\left(x_{1}\right)+\lambda_{2} \psi_{b}\left(x_{2}\right) \notin \psi_{b}(S)$ and so $\psi_{b}(S)$ is not $K_{0}$-convex for $b \in E-S$. Therefore $S \notin D\left(E_{\omega}\right)$. Now (i) and (ii) complete the proof.

REMARK 3.7. (I) Above proposition shows that the family $D^{\circ}\left(E_{\omega}\right)$ is a natural generalization to vector spaces of the concept of g.c.r.'s (cf. [1, Remark 1.6]) in the complex plane and that it offers a richer class when $E$ is a $\mathrm{K}$-i.p.s.

(II) Proposition 3.6 also establishes that all maximal subspaces and hence (cf. Proposition 3.4) their translations are members of $D^{*}\left(E_{\omega}\right)$ in a vector space but are not members of $D\left(E_{\omega}\right)$ when $E$ is a K-i.p.s.

(III) If $S \in D^{*}\left(E_{\omega}\right)$ then $E_{\omega}-S$ may not belong to $D^{*}\left(E_{\omega}\right)$. This is born out by the following example: take $E=K=C$ and let

$$
S=\{z \mid \operatorname{Im} z>0\} \cup\{z \mid-1 \leq z \leq 1\} .
$$

Then [1, Remark 1.6] $S \in D\left(\mathrm{C}_{\omega}\right)=D^{*}\left(\mathrm{C}_{\omega}\right)$ by Proposition 3.5, but $E_{\omega} S \notin D\left(\mathrm{C}_{\omega}\right)=D^{*}\left(\mathrm{C}_{\omega}\right)$.

(IV) There are sets in $D^{*}\left(E_{\omega}\right)$ whose complements in $E_{\omega}$ are also in $D^{*}\left(E_{\omega}\right)$. For example, maximal subspaces and their translations. For, if $M$ is a maximal subspace, then there exists a nontrivial linear functional $L: E \rightarrow K$ such that $M=\{x \mid L(x)=0\}$. We claim that $S=E_{\omega}-M \in D^{*}\left(E_{\omega}\right)$. Let $x, y \in E(y \neq 0)$ be arbitrarily chosen. Then $x+\rho y \in S$ if and only if $L(x)+\rho L(y) \neq 0$ provided $\rho \neq \infty$. Hence

$$
G_{s}(x, y)=K-\{-L(x) / L(y)\} \in D\left(K_{\infty}\right) .
$$

This implies by (3.4) that $S \in D^{*}\left(E_{\omega}\right)$. This shows that complements of maximal subspaces are in $D^{*}\left(E_{\omega}\right)$. The rest is established by Proposition 3.4.

(V) Thus, Remarks (II) and (III) above say that all hyperplanes and their complements in $E_{\infty}$ are members of $D^{*}\left(E_{\omega}\right)$.

\section{LUCAS' THEOREM IN VECTOR SPACES.}

In this section we prove the following main theorem on the location of the null-sets of pseudoderivatives of abstract polynomials $P: E \rightarrow K$, which generalizes to vector spaces the classical Lucas' theorem in the complex plane as well as a result due to the author [1, Theorem 3.1]. As an appliation of the main theorem we also generalize to vector spaces Marden's theorem $[4$, Corollary $(18,1)]$ on linear combinations of a polynomial and its derivatives. If $P \in P_{n}$, we shall write

$$
Z(P)=\{x \in E \mid P(x)=0\} \text {. }
$$

THEOREM 4.1. If $P \in P_{n}$ and $S \in D^{\circ}\left(E_{\omega}\right)$ such that $\omega \notin S$ and $Z(P) \subseteq S$, then

$$
Z\left(P_{h}^{\prime}\right) \subseteq S \quad \forall \quad h \in F(P) .
$$

PROOF. Let $h \in F(P)$ and $x \in Z\left(P_{h}^{\prime}\right)$. If $P_{h}^{\prime}(x)=0-P(x)$ then $x \in S$ and we are done. In case $P_{h}^{\prime}(x)=0 \neq P(x)$ we still claim that $x \in S$. On the contrary let $x \notin S$. Suppose $P \in P_{n}$ is given by (2.2). Since $h \in F(P)$ and $K$ is algebraically closed, we can write 


$$
\begin{aligned}
P(x+\rho h) & =\sum_{k=0}^{n} A_{k}(x, h) \rho^{k} \quad \forall \rho \in K, \\
& =A_{n}(x, h) \cdot \prod_{j=1}^{n}\left[\rho-\rho_{j}(x, h)\right] \quad \forall \rho \in K,
\end{aligned}
$$

where $A_{k}(x, h)$ and $\rho_{j}(x, h)$ belong to $K$ and are independent of $\rho$ such that $A_{n}(x, h)=A_{n}(0, h) \neq 0$. In the case under consideration, if we write $\rho_{j}=\rho_{j}(x, h)$, we see that $\rho_{j} \neq 0$ for all $j$ and

$$
\begin{gathered}
P(x)=A_{0}(x, h)=(-1)^{n} A_{n}(x, h) \cdot \Delta(n, n), \\
P_{h}^{\prime}(x)=A_{1}(x, h)=(-1)^{n-1} A_{n}(x, h) \cdot \Delta(n-1, n),
\end{gathered}
$$

where $\Delta(k, n)$ denotes the sum of all possible products of $\rho_{1}, \rho_{2}, \ldots, \rho_{n}$ taken $k$ at a time. Therefore

$$
\frac{P_{h}^{\prime}(x)}{P(x)}=-\sum_{j=1}^{n} 1 / \rho_{j}=0 \text {. }
$$

Since $P\left(x+\rho_{j} k\right)=0$ for all $j$, we see that $x+\rho_{j} h \in Z(P) \subseteq S$ and so $\rho_{j} \in G_{s}(x, h)=G$ (say) for all $j$. Since $S \in D^{\circ}\left(E_{\omega}\right)$ and $h \neq 0$, we observe that $G \in D\left(K_{\infty}\right)$ such that $0, \infty \notin G$ (because $\omega, x \notin S$ ) and so $\rho_{j} \neq 0$, $\infty$. Now consider the mapping $\theta_{\zeta}$ (cf. beginning of Section 2) with $\zeta=0$. Then $\theta_{\zeta}(G)$ is $K_{0}$-convex such that $0, \infty \notin \theta_{\zeta}(G)$. Since $\theta_{\zeta}\left(\rho_{j}\right)=1 / \rho_{j} \in \theta_{\zeta}(G)$, we see that

$$
(1 / n) \sum_{j=1}^{n} 1 / \rho_{j} \in \theta_{\zeta}(G)
$$

This implies that

$$
\sum_{j=1}^{n} 1 / \rho_{j} \neq 0,
$$

which contradicts (4.1). This completes the proof.

In view of Proposition 3.6, the above theorem deduces a more general version of our earlier result [1, Theorem 3.1] when $E$ is a $\mathrm{K}$-i.p.s. with $\operatorname{dim} E \geq 2$.

COROLLARY 4.2 (Zervos [8, Theorem 4, p. 360]). Let $f: K \rightarrow K$ be an $n$th degree polynomial and $f^{\prime}$ the formal derivative of $f$ (cf. Remarks 2.1 and 3.1). If $A \in D\left(K_{\omega}\right)$ such that $\omega \notin A$ and $Z(f) \subseteq A$, then $Z\left(f^{\prime}\right) \subseteq A$.

PROOF. By Remark $2.1 f \in P_{n}($ with $E-K), F(f)-K-\{0\}$ and $Z\left(f_{h}^{\prime}\right)=Z\left(f^{\prime}\right)$ for all $h \in K-\{0\}$.

Now Theorem 4.1, along with Remark 3.1 and Proposition 3.5, immediately furnish the corollary.

For $K=\mathrm{C}$, Corollary 4.2 is essentially an improved version of the (classical) Lucas' theorem (see Section 2), improvement being in the sense that we use the family $D\left(\mathbf{C}_{\omega}\right)$ of all g.c.r.'s of $\mathbf{C}_{\omega}$ instead of the classical c.r.'s as used in Lucas' theorem. Using the terminology of Lucas-sets [1, Remark 3.3], Theorem 4.1 says that every sg.c.r. $A$ of $E_{\omega}(\omega \notin A)$ is a Lucas-set for a.p.'s in vector spaces. In particular when $E$ is a K-i.p.s., the family $D^{*}\left(E_{\omega}\right) \underset{\not}{\supset} D\left(E_{\omega}\right)$ does not exhaust all Lucas-sets. This answers the two questions posed in the introduction.

Repeated applications of Theorem 4.1, together with the observations immediately preceding Remark 2.1 , give the following theorem on successive pseudo-derivatives.

THEOREM 4.3. If $P \in P_{n}$ and $S \in D^{*}\left(E_{\omega}\right)$ such that $\omega \notin S$ and $Z(P) \subseteq S$, then

$$
Z\left(P_{h}^{(k)} \subseteq S \quad \forall \quad h \in F(P), \quad 1 \leq k \leq n-1 .\right.
$$

In order to extend the above theorem to the class $\mathscr{P}_{n}^{*}$, we briefly describe the following notions and concepts, whose details can be found in [1, pp. 845-847]. A subset $M$ of $V$ is called supportable if, for each $\xi \in V-M$, there exists a lienar form $L(\equiv 0)$ on $V$ such that $L(\xi)=0$ but $L(v) \neq 0$ for all $v \in M$. If $P \in P_{n}^{*}$ is given by (2.2) and if $M$ is a supportable subset of $V$, we write

$$
\begin{gathered}
E(P) \equiv E(P, M)=\{x \in E \mid P(x) \notin M\}, \\
F^{*}(P) \equiv F^{*}(P, M)=\left\{h \in E \mid A_{n}(0, h) \in M\right\} .
\end{gathered}
$$


Since $0 \notin M$, we observe that

$$
F^{*}(P) \subseteq F(P) \quad \forall \quad P \in \dot{P}_{n}^{*} .
$$

Now we give the following more general vector-valued formulation of Theorem 4.3.

THEOREM 4.4. If $P \in P_{n}^{*}, S \in D^{*}\left(E_{\omega}\right)$ with $\omega \notin S$, and if $M$ is a supportable subset of $V$ such that $F^{*}(P) \neq \phi$ and $E(P) \subseteq S$, then

$$
E\left(P_{h}^{(k)} \subseteq S \quad \forall h \in F^{*}(P), 1 \leq k \leq n-1 .\right.
$$

PROOF. The proof is exactly the same as that of Theorem 3.9 in [1] except that the role of Theorem 3.4 in [1] is replaced by that of Theorem 4.3 of this paper.

Let us note that the hypothesis " $F^{*}(P) \approx \phi^{n}$ in the above theorem is not vacuous (cf. [1, Remark 3.8]).

For $V=K$ and $M=K-\{0\}$ the above theorem deduces Theorem 4.3 (cf. [1, Remark 3.6]) and, hence, Theorem 4.4 is a more general formulation of Lucas' theorem to vector spaces. However, Theorem 4.1 is actually the basic result employed in Theorems 4.3 and 4.4. In terms of our previous terminology [1, Remark 3.3], we see that sg.c.r.'s of $E_{\omega}$ are indeed the Lucas-sets for vector-valued a.p.'s from $E$ to $V$. Finally, we also note that Theorems 4.1, 3.4 and 3.9 in [1] are, respectively, special cases of the present Theorems 4.1, 4.3 and 4.4 when $E$ is a K-i.p.s. (cf. Proposition 3.5 (a)). Since the above-referred theorems in [1] cannot be generalized to vector spaces over nonalgebraically closed fields of characteristic zero (cf. [1', Example 4.1]), the same is true for our present theorems.

In the remainder of this section we discuss some interesting examples to suport the validity of hypotheses of our theorems here. In case $E$ is a K-i.p.s. and $S \in D\left(E_{\omega}\right) \subseteq D^{\circ}\left(E_{\omega}\right)$, this claim is supported by a number of examples discussed in [1, Section 4]. We therefore discuss examples for sg.c.r.'s in vector spaces only.

EXAMPLE 4.5. Let $E$ be an arbitrary vector space of finite or infinite dimension, with $\operatorname{dim} E \geq 2$, and consider any hyperplane $S=a+M_{0}$, where $a \in E$ and $M_{0}$ is a maximal subspace of $E$. Then $S \in D^{\circ}\left(E_{\omega}\right)$ by Remark 3.7 (II) and $\omega \notin S$. Given any fixed element $v \notin M_{0}$ (possible), every element $x \in E$ has the unique representation (cf. $[1$, p. 80])

$$
x=u+t v \text { for some } u \in M_{0}, t \in K .
$$

With this representation, let

$$
a=u_{0}+t_{0} v \text { for } u_{0} \in M_{0} \text { and } t_{0} \in K .
$$

Observe that $x \in S$ (resp. $x \in M_{0}$ ) if and only if $t=t_{0}$ (resp. $t=0$ ). If we define

$$
P_{n}(x)=\left(t-t_{0}\right)^{n} \quad \forall \quad x=u+t v \in E, \quad n=1,2,3, \ldots,
$$

we see that $P_{n}(x)=0$ if and only if $x \in S$. For $h=u^{\prime}+t^{\prime} v \in E$, we see that (for each $n$ )

$$
\begin{aligned}
P_{n}(x+\rho h) & =\left(t+\rho t^{\prime}-t_{0}\right)^{n}, \\
& =\sum_{k=0}^{n} A_{k}(x, h) \rho^{k} \quad \forall \quad x, h \in E,
\end{aligned}
$$

where the coefficients $A_{k}(x, h)-C(n, k) t^{\prime k}\left(t-t_{0}\right)^{n-k}$ are independent of $\rho$ such that $A_{n}(x, h)=A_{n}(0, h)=t^{\prime \prime} \neq 0$. This means that $P_{n} \in P_{n}$ such that $Z\left(P_{n}\right) \subseteq S$ for all $n$ and

$$
\begin{aligned}
F\left(P_{n}\right) & =\left\{h \in E \mid h \neq 0, A_{n}(0, h)=\left(t^{\prime}\right)^{n} \neq 0\right\}, \\
& =E-M_{0} \neq \phi \quad\left(\text { since } h \in M_{0} \text { iff } t^{\prime}=0\right) .
\end{aligned}
$$

We have thus shown that for every hyperplane (a member of $D^{*}\left(E_{\omega}\right)$ ), there exists (via (4.6)) infinitely many a.p.'s $P_{n} \in P_{n}(n-1,2,3, \ldots)$ satisfying the hypotheses of Theorems 4.1 and 4.3.

EXAMPLE 4.6. Let $E, V$ be vector spaces of finite or infinite dimension, with $\operatorname{dim} E \geq 2$. Consider 
the set $S=a+M_{0}$ of Example 4.5 with the same representations as given by (4.4) and (4.5). Now let $B$ be the Hamel basis for $V$ and let $\xi_{0} \in B$ be arbitrarily selected. Denote by $B_{0}$ the maximal subspace of $V$ spanned by the nonempty set $B-\left\{\xi_{0}\right\}$ and put $M=V-B_{0}$. Then $M$ is a supportable subset of $V$ such that $\xi_{0} \in M$ (cf. [1, Proposition 3.5]). Since $\xi_{0} \notin B_{0}$, every element $\xi \in V$ can be uniquely written as

$$
\xi=\eta+s \xi_{0} \text { for some } \eta \in B_{0}, s \in K,
$$

so that $\xi \in B_{0}$ if and only if $s=0$. We now choose an arbitrary (but fixed) element $\eta_{0} \in B_{0}$ and, for each $n=1,2,3, \ldots$, define (cf. (4.4) and (4.5))

$$
P_{n}(x)=\left(t-t_{0}\right)^{n} \xi_{0}+\eta_{0} \quad \forall x-u+t v \in E,
$$

where $u \in M_{0}$ and $t \in K$. Recall that $x \in S$ (resp. $\xi \in B_{0}$ ) if and only if $t=t_{0}$ (resp. $s=0$ ). Obviously, $P_{n}(x) \notin M$ if and only if $t=t_{0}$. That is (cf. (4.2) and (4.4)), $x \in E\left(P_{n}\right)$ if and only if $x \in S$. Therefore,

$$
E\left(P_{n}\right) \subseteq S \quad \forall n=1,2,3, \ldots,
$$

where $S \in D^{*}\left(E_{\omega}\right)$ such that $\omega \notin S$. Further, if $h=u^{\prime}+t^{\prime} v$ for some $u^{\prime} \in M_{0}$ and $t^{\prime} \in K$, we have (cf. (4.4))

$$
\begin{aligned}
P_{n}(x+\rho h) & =\left(t+\rho t^{\prime}-t_{0}\right)^{n} \xi_{0}+\eta_{0} \quad \forall \rho \in K, \quad x, h \in E, \\
& =\sum_{k=0}^{n} A_{k}(x, h) \rho^{k},
\end{aligned}
$$

where $A_{0}(x, h)-\left(t-t_{0}\right)^{n} \xi_{0}+\eta_{0}$ and $A_{k}(x, h)=C(n \cdot k)\left(t^{\prime}\right)^{k}\left(t-t_{0}\right)^{n-k} \xi_{0} \quad \forall \quad 1 \leq k \leq n$.

Since all these coefficients $A_{k}(x, h) \in V$ and are independent of $\rho$ such that $A_{n}(x, h)=A_{n}(0, h)=\left(t^{\prime}\right)^{n} \xi_{0} \neq 0$, it follows from the definition of a.p.'s (cf. (2.2)) that

and that (cf. (4.2))

$$
P_{n} \in P_{n}^{*} \forall n
$$

$$
\begin{aligned}
F^{*}\left(P_{n}\right) & =\left\{h \in E \mid h \neq 0, A_{n}(0, h)=\left(t^{\prime}\right)^{n} \xi_{0} \notin B_{0}\right), \text { since } M-V-B_{0}, \\
& =\left\{h \in E \mid h=u^{\prime}+t^{\prime} v, t^{\prime} \neq 0\right\} \quad \text { (due to (4.7)), } \\
& =E-M_{0} \quad(\text { due to }(4.4), \\
& \neq \phi .
\end{aligned}
$$

Consequently, in view of (4.4), (4.5), (4.7)-(4.11), we see that for every hyperplane (a member of $D^{*}\left(E_{\omega}\right)$ ) there exist vector-valued a.p.'s $P_{n} \in \mathscr{P}_{n}^{*}(n=1,2,3, \ldots)$ via (4.8) which satisfy the hypotheses of Theorem (4.4).

Finally, we apply Theorem (4.1) in a different direction to generalize to vector spaces a result due to Marden [4, Corollary $(18,1)]$ on linear combinations of a polynomial and its derivative. In what follows, $S+a$ denotes the set $\{s+a \mid s \in S\}$ where $S \subseteq E$ and $a \in E$.

THEOREM 4.7. Given $P \in P_{n}$ and $\lambda \in K$, define $R(x)=P(x)-\lambda P_{h}^{\prime}(x)$ for $h \in F(P)$. If $S \in D^{*}\left(E_{\omega}\right)$, $\omega \notin S$, such that $Z(P) \subseteq S$, then $Z(R) \subseteq S \cup(S+n \lambda h)$.

PROOF. The proof is obvious for $\lambda=0$. Therefore, we assume that $\lambda \neq 0$ and $R(x)=0$. If $x \in S$, we are done. If $x \notin S$ then by Theorem $4.1 P_{h}^{\prime}(x) \neq 0$ and $P(x) \neq 0$ (since $Z(P) \subseteq S$ ). Therefore (cf. the first equation in (4.1)),

$$
\frac{P_{h}^{\prime}(x)}{P(x)}=-\sum_{j=1}^{n} 1 / \rho_{j}=1 / \lambda \neq 0, \infty .
$$

Using the notations and steps of the proof of Theorem 4.1, with $\zeta=0$, we see that

$$
\sum_{j=1}^{n} \theta_{0}\left(\rho_{j}\right)=-1 \lambda \text {, where the } \rho_{j} \neq 0, \infty .
$$

Since $0 \notin G_{s}(x, h) \equiv G$ and $\rho_{j} \in G$, we see that, for all $j, 1 / \rho_{j}=\theta_{0}\left(\rho_{j}\right) \in \theta_{0}(G)$, where $\theta_{0}(G)$ is $K_{0}$-convex (since $G \in D\left(K_{\omega}\right)$ ). This implies that 
That is (cf. (4.12) and (4.13)),

$$
(1 / n) \cdot \sum_{j=1}^{n} 1 / \rho_{j} \in \theta_{0}(G)
$$

$$
\sum_{j=1}^{n} 1 / \rho_{j}=n / \zeta=-1 / \lambda \text { for some } \zeta \in G .
$$

Now then $x+\zeta h \in S$ (by definition of $D^{\prime}\left(E_{\omega}\right)$ ), where $\zeta=-n \lambda$. In other words, $x-n \lambda h \in S$, and hence $x \in S+n \lambda h$. This completes the proof.

COROLLARY 4.8. Given an $n$th degree ordinary polynomial $f: K \rightarrow K$ and $\lambda \in K$, define

$$
g(z)=f(z)-\lambda f^{\prime}(z) .
$$

If $A \in D\left(K_{\omega}\right), \omega \notin A$, such that $Z(f) \subseteq A$, then $Z(g) \subseteq A \cup(A+n \lambda)$.

PROOF. The proof immediately follows from Theorem 4.7 as does Corollary 4.2 from Theorem 4.1.

For $K=\mathrm{C}$, the above corollary is precisely the result due to Marden [4, Corollary $(18,1)]$. ACKNOWLEDGEMENT. This paper was supported by the Research Center Project No. Math/1404/29 of the Faculty of Science, King Saud University, Saudi Arabia.

\section{REFERENCES}

1. ZAHEER, N. On Lucas-sets for vector-valued abstract polynomials in K-inner product spaces, Cand. J. Math. 34 No. 4 (1982), 832-852.

2. WALSH, J. L. On the location of the roots of the derivative of a polynomial, C.R. Congr. Internat. des Mathematiciens. Strasbourg (1920), 339-342.

3. LUCAS, F. Proprietes géométriques des fractions rationelles, C. R. Acad. Sci. Paris 77 (1874), 431-433; ibid. 78 (1874), 140-144; ibid. 78 (1874), 180-183; ibid. 78 (1874), 271-274.

4. MARDEN, M. Geometry of Polynomials, rev. ed., math. Surveys, No. 3, Amer. Math. Soc., Providence, R.I., 1966.

5. HILLE, E. and PHILLIPS, R. S. Functional Analysis and Semi-groups, rev. ed., Amer. Math. Soc. Colloq. Publ., Vol. 31, Amer. Math. Soc., Providence, R.I., 1957.

6. TAYLOR, A. E. Additions to the theory of polynomials in normed linear spaces, Tôhoku Math. L 44 (1938), 302-318.

7. ZAHEER, N. Null-sets of Abstract Homogeneous Polynomials in Vector Spaces, Doctoral thesis, University of Wisconsin, Milwaukee, Wisconsin, 1971.

8. ZERVOS, S. P. Aspects modernes de la localization des zéros des polynômes d'une variable, Ann. Sci. École Norm. Sup (3) 77 (1960), 303-410.

9. ZAHEER, N. On polar relations of abstract homogeneous polynomials, Trans. Amer. Math. Soc. 218 (1976), 115-131.

10. BOURBAKI, N. Éléments de Mathématique, XIV. Part 1, Les Structures Fondamentales de l'analyse. Livre II: Algébre, Chap. VI: Groupes et Corps Ordonnés, Actualites Sci. Indust., 1179, Hermann, Paris, 1952.

11. HÖRMANDER, L. On a theorem of grace, Mth. Scand 2 (1954), 55-64.

12. VANDER WAERDEN, B. L. Algebra, Vol. I, 4th ed., Die Grundlehren derMath. Wissenschaften, Band 33, Springer-Verlag, Berlin, 1955, English Tran., Ungar, New York, 1970.

13. WILANSKI, A. Functional Analysis, Blaisdell, New York, 1964.

14. MARDEN, M. A generalization of a theorem of Bôcher, SIAM J. Numer. Anal. 3 (1966), 269-275.

15. MARDEN, M. On composite abstract homogeneous polynomials, Proc. Amer. Math. Soc. 22 (1969), 28-33.

16. ZAHEER, M. On composite abstract homogeneous polynomials, Trans. Amer. Math. Soc. 228 (1977), 345-358.

17. ZAHEER, N. On generalized polars of the product of abstract homogeneous polynomials, Pacific J. Math. 74, No. 2 (1978), 535-557.

18. ZAHEER, N. and ALAM, M. Zeros of polar-composite polynomials in algebraically closed fields, Proc. London Math. Soc. (3) 40 (1980), 527-552.

19. BERBERIAN, S. K. Lectures in Functional Analysis and Operator Theory, Graduate Texts in Mathematics, Vol. 15, Springer-Verlag, New York, 1974. 


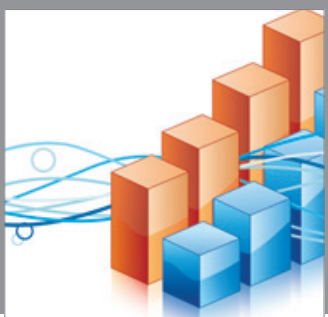

Advances in

Operations Research

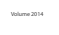

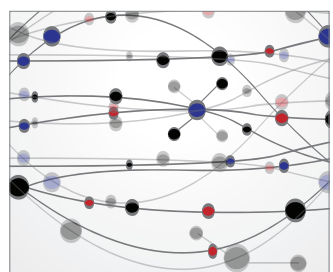

\section{The Scientific} World Journal
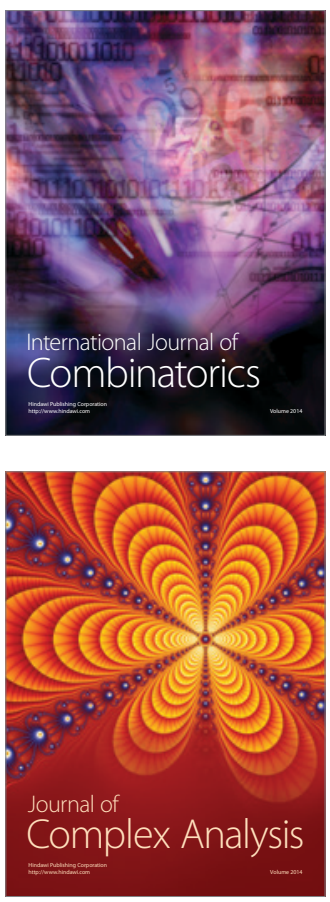

International Journal of

Mathematics and

Mathematical

Sciences
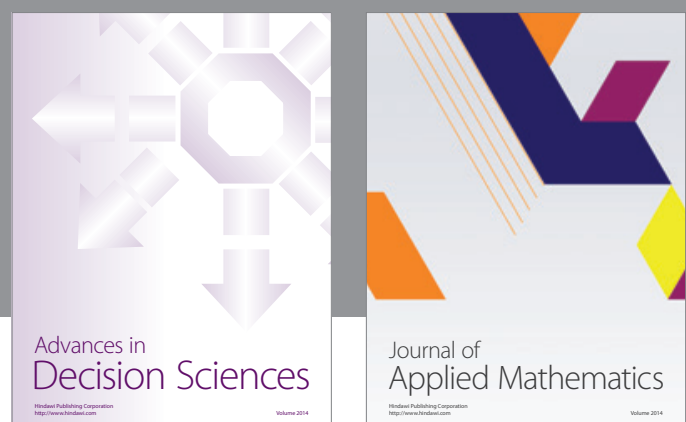

Journal of

Applied Mathematics
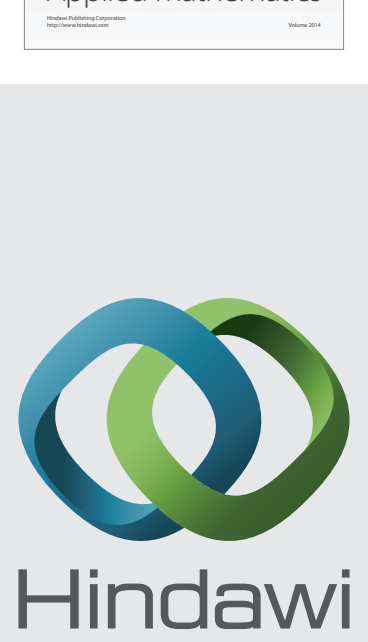

Submit your manuscripts at http://www.hindawi.com
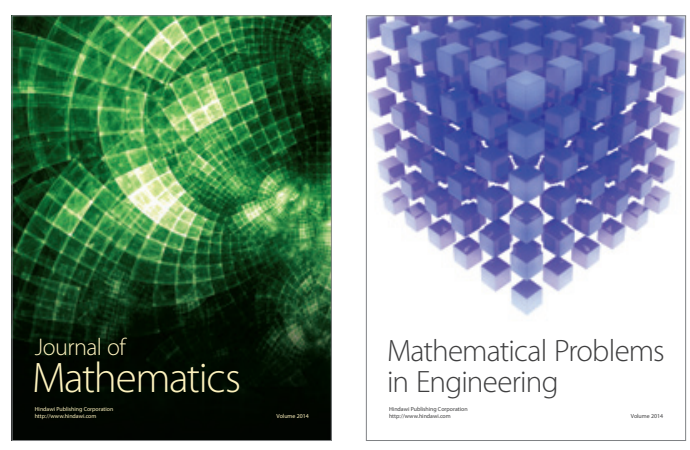

Mathematical Problems in Engineering
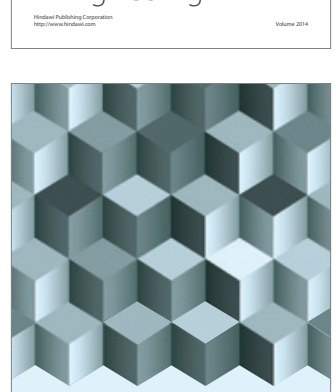

Journal of

Function Spaces
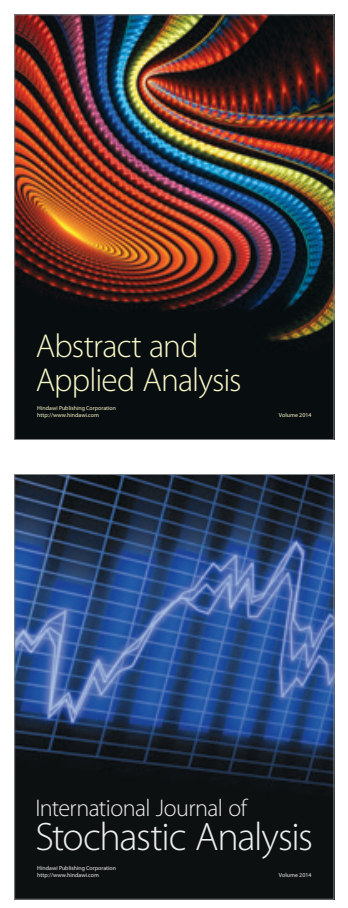

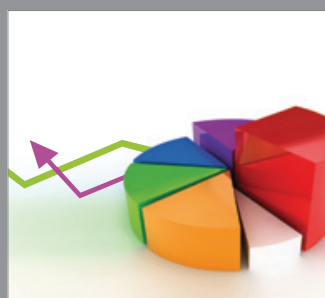

ournal of

Probability and Statistics

Promensencen
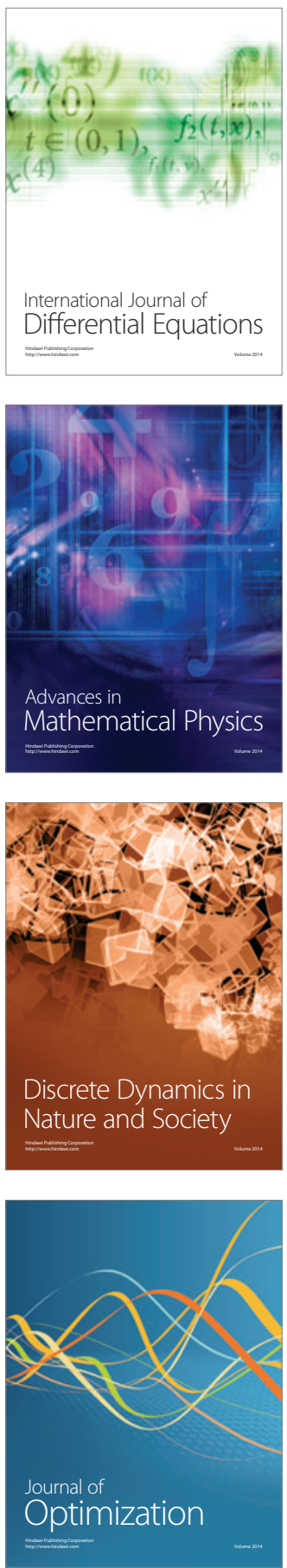